\title{
Integrative modeling of structure and dynamics of macromolecules based on SAXS profiles and cross-linking mass spectrometry
}

\author{
Dina Schneidman \\ The Hebrew University of Jerusalem, Jerusalem, Israel; \\ dina.schneidman@mail.huji.ac.il
}

Proteins generally populate multiple structural states in solution. Transitions between these states are important for function, such as allosteric signaling and enzyme catalysis. Structures solved by X-ray crystallography provide valuable, but static, atomic resolution structural information. In contrast, cross-linking mass spectrometry (XLMS) and small angle X-ray scattering (SAXS) datasets contain information about conformational and compositional states of the system. The challenge lies in the data interpretation since the crosslinks in the data often comes from multiple structural states. We have developed a novel computational method that simultaneously uncovers the set of structural states that are consistent with a given dataset (XLMS or SAXS). The input is a single atomic structure, a list of flexible residues, and an experimental dataset. The method finds multi-state models (models that specify two or more coexisting structural states) that are consistent with the data. The method was applied on multiple SAXS and XLMS datasets, including large multi-domain proteins and proteins with long disordered fragments. The applicability of the method extends to other datasets, such as 2D class averages from Electron Microscopy, and residual dipolar couplings.

Keywords: protein dynamics, multi-state modeling 